\title{
Mapping-Based Exchange of Models Between Meta-Modeling Tools
}

\author{
Heiko Kern Fred Stefan \\ University of Leipzig \\ Business Information Systems \\ Augustusplatz 10, 04109 Leipzig, Germany \\ \{kern, stefan\}@informatik.uni-leipzig.de
}

\author{
Vladimir Dimitrieski Milan Čeliković \\ University of Novi Sad \\ Faculty of Technical Sciences \\ Trg Dositeja Obradovića 6, 21000 Novi Sad, Serbia \\ \{dimitrieski, milancel\}@uns.ac.rs
}

\begin{abstract}
The exchange of models between meta-modeling tools is an important requirement. Tools often only cover a certain task in the development process. The exchange of models between different tools is necessary for covering a complete development process. Besides the aspect of cooperation, exchange of models also enables the replacement of old tools by new tools that better fit the customer's needs. In order to avoid the vendor lock-in effect, model exchange allows the reuse of existing models. In this paper, we focus on the problem of model exchange and present an mappingbased approach between different meta-modeling tools. The approach is centered around a declarative mapping language with a graphical notation and a solution for connecting different metamodeling tools. We apply our approach to exchange models between MetaEdit+ and Microsoft Visio.
\end{abstract}

Categories and Subject Descriptors D.2.12 [Interoperability]: Data mapping; D.2.6 [Programming Environments]: Graphical environments

\section{General Terms Design, Languages, Experimentation}

Keywords migration, interoperability, mapping, code generation, model transformation, meta-modeling

\section{Introduction}

Models play an important role in Domain-Specific Modeling [6] and other related development disciplines. Generally, models represent a system in an abstract way, improve the understanding of a system, and facilitate the communication between different stakeholders. The creation of models is the result of a modeling process which is supported by a modeling tool. A special class of these modeling tools are meta-modeling tools. In addition to providing a user with a set of pre-defined modeling languages, meta-modeling tools provide a mechanism for the specification of new modeling languages. Modeling concepts of these languages are specified in a form of meta-models. Examples of meta-modeling tools are: MetaEdit+ [6], Generic Modeling Environment [12], and Microsoft Visio [2].

An important requirement for modeling tools, including metamodeling tools, is the interoperability with other tools. In the context of this paper, interoperability is defined as the ability of two or more tools to exchange models or meta-models. Additionally, these exchanged models and meta-models must be usable in these tools. Often, tools support a specific task in the development process. Therefore, a successful application of the whole development process depends heavily on the degree of interoperability between the tools used. Besides the cooperation of tools, evolution of a tool landscape is an important aspect. As the software industry constantly evolves, modeling tools also evolve and old ones are being replaced by new tools that better fit the customer's needs. In order to avoid the vendor lock-in effect, interoperability between tools is necessary and enables the reuse of existing models between tools from different vendors.

The interoperability between meta-modeling tools is not widely supported [8]. There is no suitable model exchange approach that takes meta-models into consideration. In this paper, we address this lack of interoperability between meta-modeling tools and present an approach to realize the exchange of models in consideration of their meta-models. The goal of this approach is to allow an efficient and user-oriented import and export of models in tools currently used in the industry.

The paper is structured as follows. In Section 2, we introduce the problem of model exchange between meta-modeling tools. In the same section we derive requirements for our approach. In Section 3, we present our solution in detail. Afterward, we present a use case in Section 4 and a evaluation of the approach in Section 5. We discuss related work in Section 6 and conclude this paper in Section 7 with a summary and suggestions for future work.

\section{Purpose and Requirements}

In this paper we address the exchange of models between metamodeling tools. In contrast to simple modeling tools, the exchange of models between meta-modeling tools is a challenging task because the exchange must consider the heterogeneity of the metamodels. A study about interoperability between meta-modeling tools [8] shows that currently there are no suitable approaches allowing this kind of model exchange. The majority of metamodeling tools provides an export of meta-models and models in a specific serialization format, e.g. Extensible Markup Language (XML) with a predefined schema, XML Metadata Interchange (XMI), or Graph eXchange Language (GXL). These solutions are unsatisfactory because a serialization format cannot solve the problem of having different meta-model elements.

The tool approach that we want to present should exchange models in dependency of their meta-models between different tools. We determine that the models of the source and target tool implement the same language by using meta-models. These metamodels can have heterogeneity stemming from the tool-specific meta-modeling language and different ways to express a language. Although an automatic creation of the language in the target tool is possible, it is unsatisfactory as some of the particularities of the target meta-modeling tool are lost. Hence, we assume that the language is already manually specified by a language engineer in both tools. Beside this assumption, the source and target models must be 


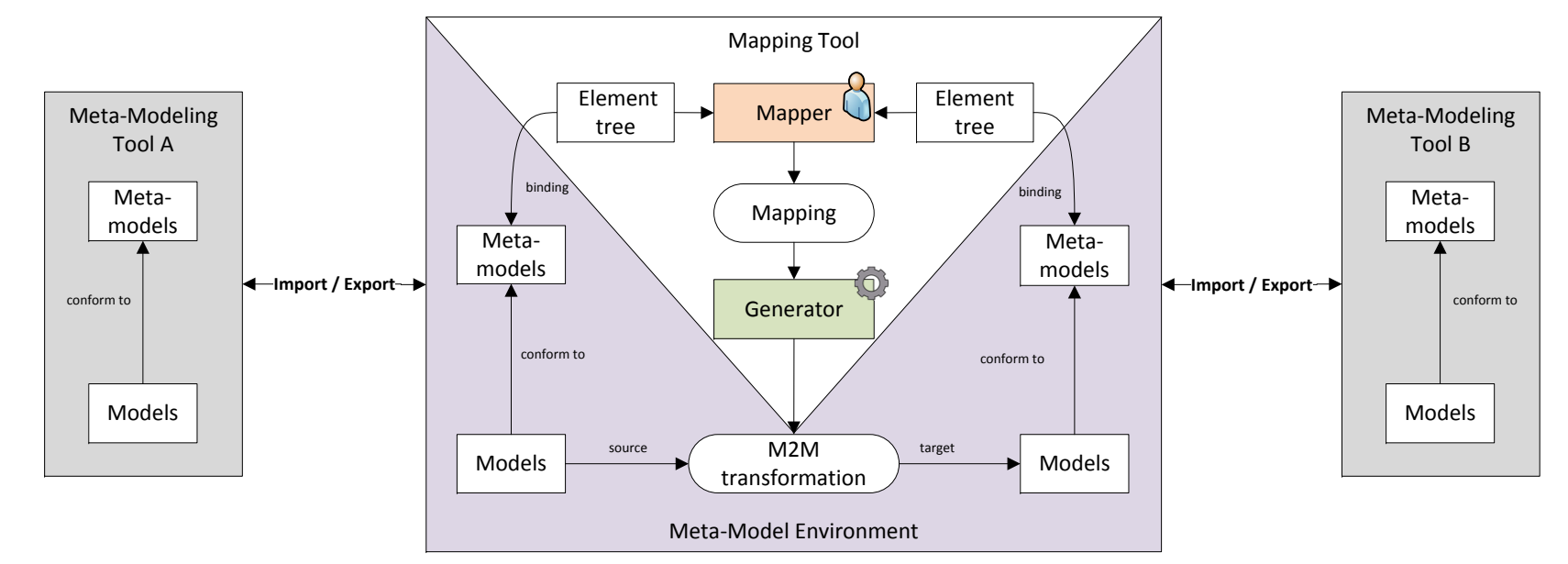

Figure 1. Overview of the mapping-based model exchange

in an isomorphic relationship after the exchange. The approach we propose uses model transformation concepts from Model-Driven Engineering. Beside the defined purpose, the approach and the appropriate tooling must satisfy the following requirements:

(i) Graphical and declarative language. The mapping approach must comprise a declarative language with a graphical notation for specification of correspondences between source and target metamodels. The primary target audience of the mapping approach are users of modeling tools. These users are familiar with graphical modeling usually used in these kind of tools. Hence, we assume that a graphical mapping language fits this group's needs better than a textual transformation language. Furthermore, we assume that the correspondence specification using a graphical language suits the exchange process better than the manual implementation of transformation code. The declarative nature of the language allows the abstraction from execution details. We want to hide these execution details from the user as the focus should be solely on the specification of correspondences between language concepts.

(ii) Structural independence. The model exchange approach must be independent from the structure of meta-models and models that have to be exchanged. The structural independence allows a broad application of the exchange approach and enables the connection of different meta-modeling tools. To support this requirement, a generic algebraic structure has to be utilized in the approach. All of the exchanged meta-models should be transformed to this generic structure before the specification of correspondences.

(iii) Operational independence. The mapping approach itself must be independent of the transformation execution environments which realize the model exchange. Therefore, the mapping language concepts must be independent of specific transformation execution environments. Using such a language, a user would be able to use the approach on two meta-models from different metamodeling tools. Based on the correspondences, an executable transformation is created through an automatic generation process.

\section{Mapping-Based Model Exchange Approach}

Our approach for model exchange can be divided into several steps. In Figure 1 we present an overview of this approach. The first step is the import of meta-models from meta-modeling tools. For this task, we use M3-Level-based Bridges (M3B) [7, 9, 10]. A M3B transforms meta-models into an intermediate meta-model environment. After that, a binding component creates a generic tree structure for representing meta-models. Although the imported meta-models implement the same language, there is still some heterogeneity be- tween their elements. For instance, elements with the same meaning may have different names or relationships can be specified in a different way. In order to overcome this heterogeneity, the user defines a mapping containing correspondences between different elements. In the next step, a generator iterates over specified mappings and produces an executable model-to-model transformation. The generator is specific to the pair of source and target metamodeling tools as the transformation must be able to read source models and produce valid target models. The generated model-tomodel transformations are defined against the imported source and target meta-models. The final step is the execution of the transformation including the exchange of models by using a M3B.

\subsection{Import of Meta-Models}

An important aspect of the mapping tool is the exchange of model data (models and meta-models) between our mapping tool and connected meta-modeling tools. In the first step of the approach, meta-models are imported in our mapping tool and in the last step models are exchanged between the different meta-modeling tools. For the import and export of modeling data, we use the M3B approach. Generally this approach allows a transformation of models and meta-models between different meta-modeling environments. Furthermore, the M3B concerns technical and meta-modeling toolspecific heterogeneity. An M3B comprises at least two transformations, one at meta-model level and one at model level. The meta-model transformation transforms meta-models between metamodeling environments. Both meta-models are in an isomorphic relationship necessary for the latter transformation at the model level. The model transformation reads models of the source metamodeling environment and creates models that are to be used in the target meta-modeling environment. The created target models must conform to the meta-model prior imported by a M3B. Based on the imported meta-models, we create a general tree representation.

\subsection{Representation of Meta-Models}

Our mapping approach aims to provide a generic mechanism for specifying mapping regardless of the meta-modeling tool. For this reason, we provide a generic tree representation of meta-models. A binding component reads the meta-models and creates a tree structure of the meta-model elements.

Concepts of the generic tree structure are presented in Figure 2. Each meta-model in a mapping is represented by an element container (ElementContainer). Each element container has a name corresponding to the name of an imported meta-model. 


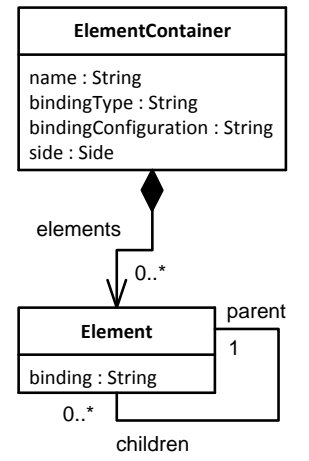

Figure 2. Meta-model of the generic tree structure

This name allows the differentiation between various imported meta-models. Furthermore, an element container has a binding type (bindingType) and a binding configuration (bindingConfiguration). The binding type attribute determines the selection of a binding component and later the generation of a model transformation. During the binding process, the binding component transforms a metamodel into a generic tree structure. The tree elements have a reference to the native meta-model elements. This reference is necessary during the generation process of the model-to-model transformation in order to get the native meta-model elements. Furthermore, the binding type is necessary for reading the binding configuration. The binding configuration in turn specifies binding details for the element container. A value of the side parameter (side) determines if the element container has a role of source or target container in a mapping.

Each element container comprises zero or more elements ( $\mathrm{El}$ ement). Each element has a binding string (binding) and a name (name). The name attribute corresponds to the name of an original element from an imported meta-model. The path to the original element is stored in the binding string. The format of the binding string depends on the technology of the original element.

\subsection{The Mapping Language}

The mappings between two element trees are expressed by a mapping language. In Figure 3, we present the abstract syntax of this mapping language. The root element of a mapping description is a mapping container (MappingContainer). A mapping container comprises element containers, zero or more links (Link) and nodes (Node). An element container, introduced in the prior Section 3.2, contains elements that can be part of a mapping. Each mapping container has at least one source and one target element container. Each mapping is represented with a selector (Selector) linked to source and target elements. Based on the number of source and target elements participating in a mapping, we classify selectors as one-to-one (OneToOne), one-to-many (OneToMany), many-toone (ManyToOne), many-to-many (ManyToMany), and zero-toany (ZeroToAny). Selectors have a name (name property) for their unique identification in a mapping container, and a select expression (selectExpression property) for specifying a filter on all source elements. Each selector is connected to source and target elements using links (Link). As each link connects an element to a selector, conditions relating to the single element are specified at the level of the link (condition property).

Mappings can depend on other mappings. The dependency of mappings results from the parent-child relation of the element tree from the source or target structure. If all elements that are being mapped have no parent elements in the tree, the mapping is considered to be a root mapping. On the other hand, if one of the participating elements has a parent element in the tree, the mapping is considered to be a dependent mapping.

A selector can use one function (Function) that performs calculations on source elements participating the mapping. The result of a function is to be mapped to a target element. As a function represents a reusable unit of code, it is specified at the level of a mapping container and can be used by multiple selectors. For each function usage (SelectorFunction) in a selector, arguments have to passed (ArgumentAssignment) to the function. Passing an argument from a selector to a function requires a specific source element, identified by its link (linkId property), to be passed to a specific parameter, identified by the ordinal number in its function (parameterNumber property). Source elements can be passed as input parameters, while target elements get their value from output elements. A type of an argument assignment is specified by the parameterType property of the ArgumentAssignment concept.

The only selector not requiring a source element to be mapped to a target element is the zero-to-any selector. This selector provides creation of any number of target elements when corresponding elements do not exist in source meta-model. Target elements can be created without setting values of their attributes. However, a result of a function without parameters or a constant value (ConstantValue) can be assigned to the target element attributes.

\subsection{Implementation}

Our mapping tool allows the specification of mappings between arbitrary meta-modeling tools. For this purpose, we have chosen the Eclipse Modeling Framework (EMF) [18] as a mediator. This meta-modeling environment offers basic meta-modeling concepts in comparison to other environments [11]. These basic concepts allow the emulation of concepts from other meta-modeling environments. Furthermore, EMF provides various model processing tools that suit our needs and ease the development process. All of the meta-models and models are imported into EMF by using an M3B. There are existing implementations of M3B which allows the connection of different meta-modeling tools and EMF. Binding components are implemented in Java. The graphical editor for mapping specification is implemented by two different technologies. We use the Sirius framework [17] for the rapid prototyping of the graphical editor. Sirius allows us to try out new language concepts by automatically generating the editor based on the mapping language. The drawback of such editor is its hard customization. On the other hand, we use the Standard Widget Toolkit (SWT) [19]. The SWT-based editor has to be developed manually without automatic generation. However, it provides us with the full control over the features and customization of the editor. Finally, code generators are developed in Xtend [21].

\section{Case Study: EPC Model Exchange}

In this section, we present a case study to demonstrate a complete exchange process provided by our tool. The case study shows the exchange of models between MetaEdit+ and Microsoft Visio. The models in this example conform to Event-Driven Process Chain (EPC) language.

The exchange process starts with the import of EPC metamodels from both meta-modeling tools. As our approach uses EMF as the mediator, both meta-models are first transformed by an M3B into EMF. The outputs of the transformations are the MetaEdit-EPC and Visio-EPC meta-models conforming to Ecore. Afterwards, the binding components create tree representations of both meta-models. In Figure 4, we present a mapping between the imported EPC meta-models. The meta-model from MetaEdit+ is presented on the left-hand side and the Visio meta-model is depicted on the right-hand side in this screenshot. The presented tree view comprises meta-model elements, meta-metamodel elements, 


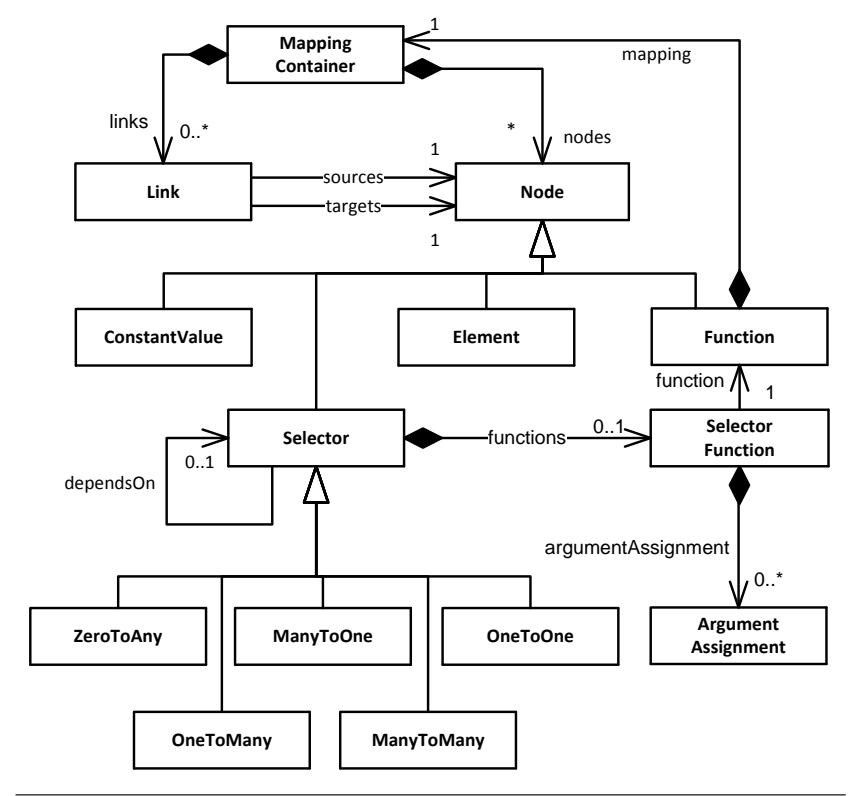

Figure 3. Meta-model of the mapping language

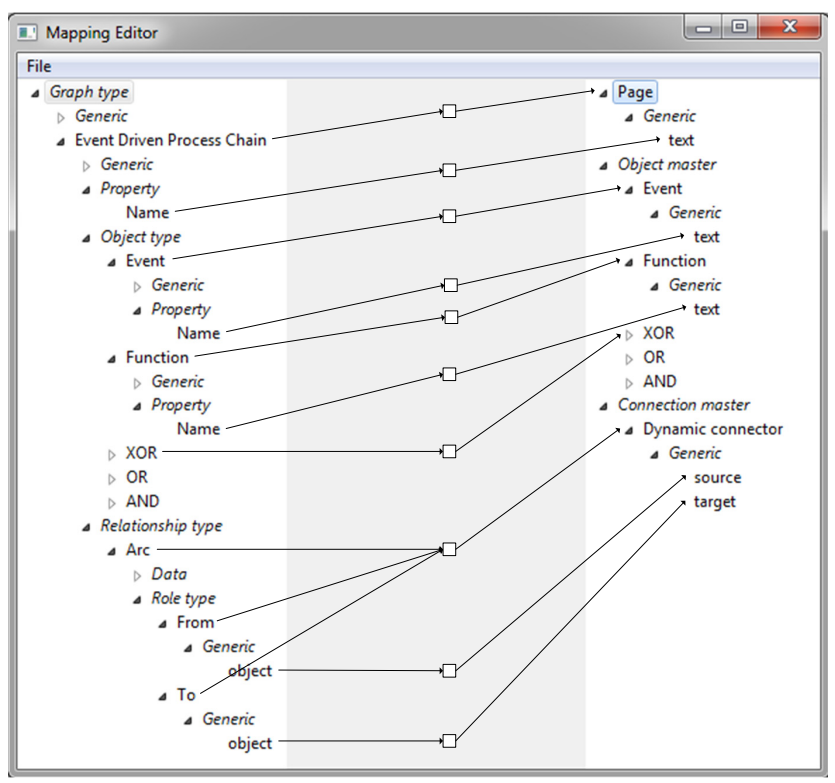

Figure 4. Mappings between EPC meta-models and generic elements. The meta-metamodel elements are presented in italics which helps in organizing different meta-model elements. The generic elements are independent of a concrete meta-model. Generic elements are not defined by the language engineer during the meta-model specification. The generic elements are given by the meta-modeling tool. For instance, in Visio each shape has a text property which is often used as label in a shape.

The central part of Figure 4 depicts mappings with their selectors and links (cf. mapping language). For instance, the MetaEdit+ "Event Driven Process Chain" graph type is mapped to a Visio page as Visio does not support a graph type concept. The name property of this graph type is mapped to the generic element "text" of a Visio page. This property mapping is considered to be a child mapping of the graph type mapping. Both mappings are one-to-one mappings. Next, the object types are mapped into the corresponding object masters. The relationship type "Arc" from MetaEdit+ is mapped to the Visio connection master "Dynamic connector". This mapping is a many-to-one mapping because the mapping needs to include arc role types. The referenced object of the "From" role type is mapped to "source" and the object of the "To" role type is mapped to "target" of "Dynamic connector".

After defining the mapping, a generator creates the model-tomodel transformation. Generally we can distinguish two parts of the transformation: a meta-model independent (MMI) and a metamodel dependent transformation (MMD). A MMI-transformation implements rules that are independent of certain modeling languages. These rules only depend on the meta-modeling tools that are participating in the exchange. Once implemented, these rules can be reused for all transformations between languages specified in these tools. An example of a MMI-transformation rule is a transformation of symbol coordinates between MetaEdit+ and Visio. An object in MetaEdit+ may be related to a symbol that has a position on a diagram. Similarly, each shape in Visio also has a position on a page. The MMI-transformation rule is used to transform the MetaEdit+ symbol position into the Visio shape position.

The second part (named MMD-transformation), concerns the mapping of the language-specific concepts. These transformation rules depend on particular modeling languages and not directly on meta-modeling tools. Therefore, the generation of this trans- formation depends on the previously defined mapping rules. In this case study, the generator produces an Epsilon Transformation Language (ETL) transformation. The generation of other transformation languages is also possible by simply invoking a different generator. In Listing 1, we present a snippet of the MMDtransformation code. Due to space limitations, we do not present the MMI-transformation. The first rule in Listing 1 corresponds to the first mapping rule that creates a Visio page for each MetaEdit+ EPC graph. The name of a MetaEdit-EPC graph is assigned to the name of a Visio page. Similar to this, the next rules represent a transformation of model elements that are instances of the Event, Function, XOR, and Arc concepts. Figure 5 shows a MetaEdit+ EPC model as input and the resulting Visio EPC model.

\section{Evaluation}

In the previous section we presented one application of our mapping tool. In addition to the EPC case, we applied our tool to the exchange of models expressed in other modeling languages, such as Unified Modeling Language (UML) class diagram and Business Process Modeling Notation (BPMN). Based on these applications, we evaluate the approach with regards to the following criteria:

(i) Completeness and quality. The mapping tool works well with simple meta-models (e.g. EPC meta-model) and more complex meta-models (e.g. UML class diagrams and BPMN). Organizing meta-model elements as element trees is suitable for the representation of any meta-model. The mapping language provides users with enough concepts to express all needed mappings and to generate an executable model transformation between the participating meta-models. The end results of the migration process are satisfactory as the created target models fully correspond to the source models. We identified one weakness which is not directly related to our mapping approach but rather on the import and export of meta-models and models in our mapping tool. The quality of the exchange process depends heavily on the quality of the imported and exported model data. The used M3B approach has proved as suitable, but in the end the quality depends on the model interfaces provided by the connected meta-modeling tools.

(ii) Usability. One of the main elements of our tool influencing the usability aspect is its user interface. We distinguish between two 


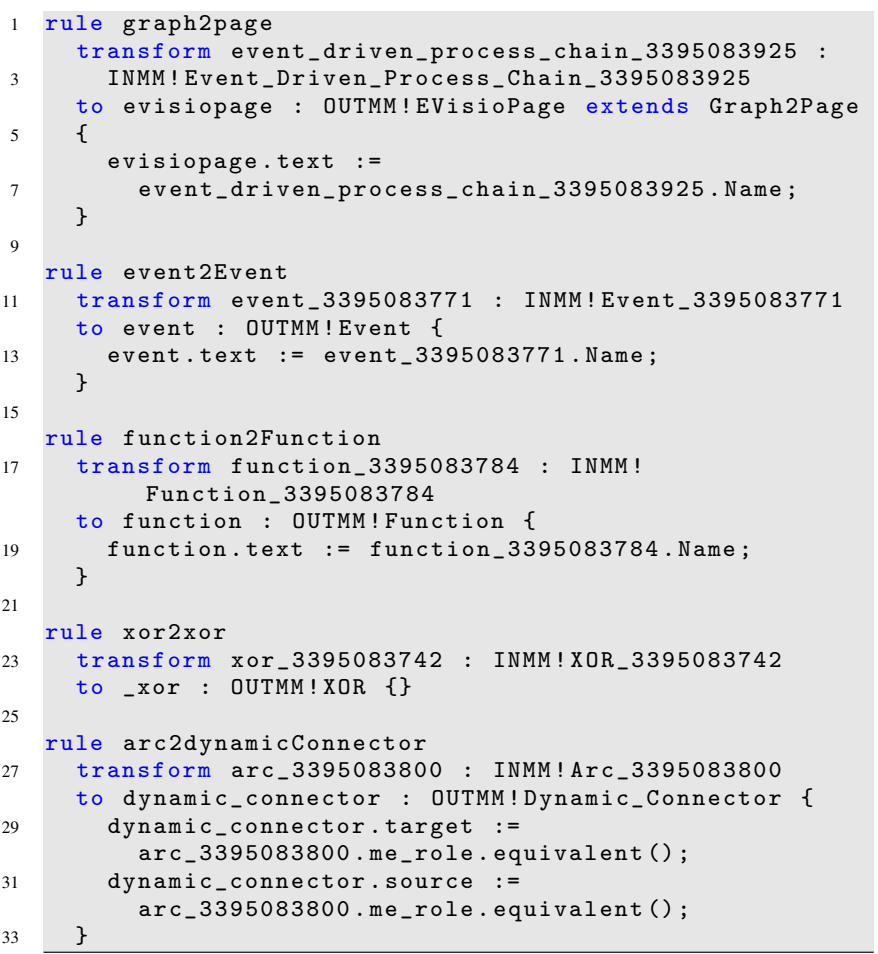

Listing 1. Snippet of the generated ETL transformation

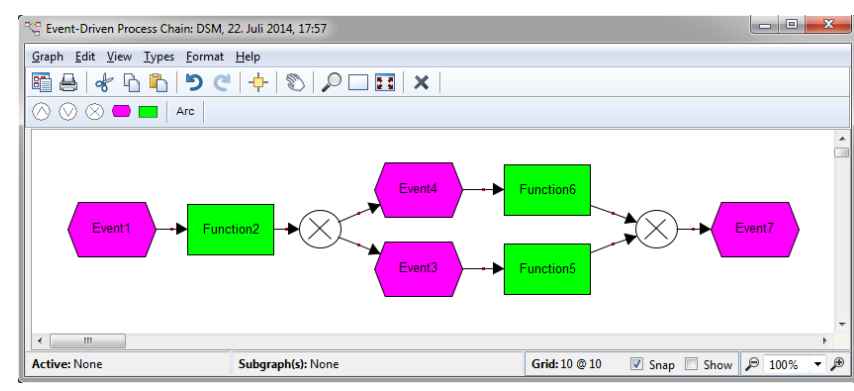

(a) Source: MetaEdit+

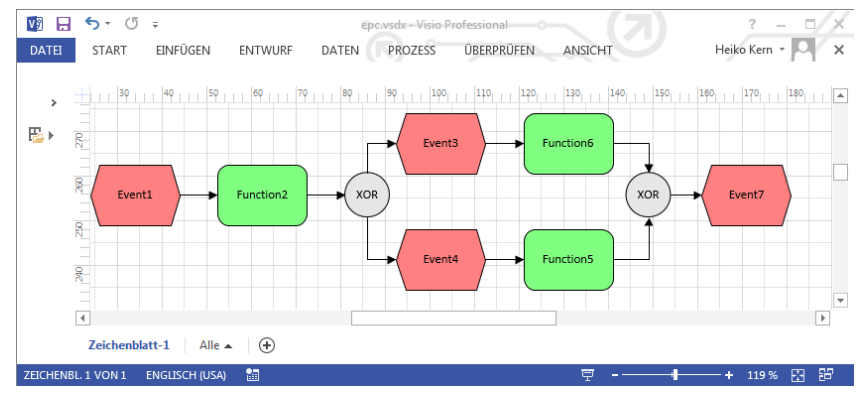

(b) Target: Microsoft Visio

Figure 5. EPC model example

parts of the user interface: the graphical interface for the specification of mappings and the tree representation of meta-models. The graphical notation of the mapping fits the need of users who are familiar with graphical modeling tools well. The graphical mapping is intuitive and enables a good overview of the mapping. In the examples (EPC, UML, and BPMN) the mapping is easy to interpret. Nevertheless, we find that the graphical representation of complex mappings could be confusing due to the amount of lines. The second part relates to the representation of the meta-models as tree structures. The tree representation is a suitable view on metamodels as part of a mapping. The tree representation focuses on the meta-model concepts and abstracts from unnecessary details, e.g. references between meta-model elements. The additional items in the tree, e.g. italic tree items in Figure 4, give a user additional information and allow easier mapping specification. Our mapping tool also improves the usability of the exchange process in general. Instead of programming a model transformation, the tool generates the executable model transformation. Regarding a comparison of Figure 4 and Listing 1, we assume that through the abstraction, the usability of our mapping approach is better for modeling tool users than it is the case when they are programming with textual model transformation.

(iii) Adaptability and expandability. Generally, the mapping tool supports the exchange between meta-modeling tools implementing a three-level model hierarchy. The meta-modeling tools must allow the export of meta-models and the import/export of models. The representation of meta-models as element trees allows the import of meta-models from various meta-modeling tools. This way, our tool satisfies the requirement for structural independence (cf. Section 2). For each meta-modeling tool a special binding component has to be developed. A binding component can be easily added to our mapping tool in the form of a plug-in. Furthermore, different views can be created on top of the element tree. Currently, we have one implementation including a tree view (cf. case study) and a custom graphical view. Due to space limitations the custom graphical view is not presented in this paper. The mapping itself is represented as an EMF model. This allows the creation and addition of new code generators for different transformation execution environments. This way, our tool satisfies the requirement for operational independence (cf. Section 2).

\section{6. $\quad$ Related Work}

We found several mapping approaches and environments proposed in literature. Wimmer proposes in [20] an approach to model-based tool integration in the context of the ModelCVS project. Similarly to our approach, Wimmer proposes three steps: importing models, specifying mappings, and executing the mappings. This approach however heavily depends on the EMF technical space. Mapping operators are directly based on the notions and concepts from the EMF technical space. This solution also proposes execution of the mappings by executing colored Petri nets while our approach generates executable transformation code in a desired transformation language.

In [1], authors present the ATLAS Model Management Architecture (AMMA). AMMA is a set of tools and languages that can be used in the process of tool integration. The central part of AMMA is a mapping language and a tool named ATL Model Weaver (AMW) [13]. AMW provides an extendable core language for specifying platform independent transformations. Authors of the paper argue that for a tool integration process, a specific language should be derived from the core language in order to cover the specific need of that process. However, we feel that this could be burdensome for the users of such a tool as for each integration scenario they need to create new concepts. Our goal is to provide a simple yet powerful language that can be used regardless of the tools being integrated.

Several other mapping approaches can be found in literature, such as Clio [15], Rondo [14], RDFT [16], and the UML-based approach presented in [5]. All of these approaches focus on the integration of certain technical spaces or languages, such as, XML, 
Relational Databases or UML. However, none of these approaches focus on the integration of (meta-)modeling tools.

The mapping language in our tool can be regarded as a graphical transformation language used for the specification of platform independent transformations. Bollati in [3] presents an approach to modeling transformations using a composition of a graphical modeling language and natural language to specify platform independent transformation models (PIMs). The author proposes a definition of PIMs at a high level of abstraction while covering a relatively small amount of details. In contrast to that, we provide in our approach a meta-model of PIMs that allows a modeler to specify transformations with a significantly greater number of details. In much greater detail, several other graphical transformation languages are surveyed in [4]. However, these languages are used for platform specific transformation specification. They are provided with their own execution environment and thus are often limited in the type of meta-models that may be transformed. The languages described in [4] are mostly limited to the EMF technical space.

\section{Conclusion}

In this paper we presented a mapping-based approach to exchange models between different meta-modeling tools. The approach has three phases. In the first phase, meta-models are imported from the meta-modeling tools. For the import of the meta-models, we use M3-Level-based Bridges. Imported meta-model elements are represented in the form of the generic element tree structure. In the second phase, a user can use a declarative language with a graphical notation to specify mappings between source and target metamodels. The final phase of the approach comprises the generation of executable model-to-model transformations.

In order to evaluate the approach, we presented an application scenario for our mapping tool. The case study concerns the exchange of EPC models between MetaEdit+ and Microsoft Visio. The results of the evaluation show that the mapping tool allows a user to generate complete executable transformations. We also showed, that the generic tree structure and the mapping language concepts are sufficient for easy and efficient mapping specification. From the viewpoint of adaptability and extendability, connections to other meta-modeling tools and generators could be easily add to our tool in the form of plug-ins.

One direction of future work is finding ways to improve the user interface of the mapping tool. In case of large meta-models, a mapping diagram could get overcrowded with links and selectors. This could be improved by using a tabular view of mappings with less graphical lines between elements. Furthermore, imported meta-models often have repeating structures. To assist the user in specifying new mapping models, we want to reuse existing mappings. Future research will be focused on developing algorithms that choose appropriate existing mapping to be applied to new situations.

\section{Acknowledgments}

Research presented in this paper was supported by the German Exchange Service and Ministry of Education, Science and Technological Development of Republic of Serbia as part of the bilateral project "Discovering Effective Methods and Architectures for Integration of Modeling Spaces with Application in Various Problem Domains", 2014 - 2015.

\section{References}

[1] J. Bézivin, F. Jouault, P. Rosenthal, and P. Valduriez. Modeling in the large and modeling in the small. In Model Driven Architecture, page 33-46. Springer, 2005.
[2] B. Biafore. Visio 2007 Bible. Wiley Publishing, April 2007. ISBN 978-0470109960.

[3] V. A. Bollati. MeTAGeM: Entorno de Desarrollo de Transformaciones de Modelos Dirigido por Modelos. PhD thesis, Universidad Rey Juan Carlos, 2010.

[4] V. Dimitrieski, I. Lukovic, S. Aleksic, M. Celikovic, and G. Milosavljevic. An Overview of Selected Visual M2M Transformation Languages. In International Conference on Information Society Technology and Management, 2014.

[5] J. H. Hausmann and S. Kent. Visualizing model mappings in UML. In Proceedings of the 2003 ACM symposium on Software visualization, page 169-178. ACM, 2003.

[6] S. Kelly and J.-P. Tolvanen. Domain-Specific Modeling: Enabling Full Code Generation. Wiley-IEEE Computer Society, March 2008. ISBN 978-0-470-03666-2.

[7] H. Kern. Interchange of (Meta)Models between MetaEdit+ and Eclipse EMF using M3-Level-Based Bridges. In J. Gray, J. Sprinkle, J.-P. Tolvanen, and M. Rossi, editors, Proceedings of 8th OOPSLA Workshop on Domain-Specific Modeling, pages 14-19, 2008.

[8] H. Kern. Study of Interoperability between Meta-Modeling Tools. In Proceedings of the Third Workshop on Model-Driven Approaches in System Development (MDASD) at Federed Conference Science and Information Systems, 2014. to appear.

[9] H. Kern and S. Kühne. Model Interchange between ARIS and Eclipse EMF. In J.-P. Tolvanen, J. Gray, R. Matti, and J. Sprinkle, editors, Proceedings of 7th OOPSLA Workshop on Domain-Specific Modeling, pages 105-114, 2007. ISBN 78-951-39-2915-2.

[10] H. Kern and S. Kühne. Integration of Microsoft Visio and Eclipse Modeling Framework Using M3-Level-Based Bridges. In C. Hein, T. Ritter, and M. Wagner, editors, Proceedings of Second Workshop on Model-Driven Tool and Process Integration (MDTPI) at ECMFA, CTIT Workshop Proceedings, pages 13-24. University of Twente, June 2009.

[11] H. Kern, A. Hummel, and S. Kühne. Towards a Comparative Analysis of Meta-Metamodels. In Proceedings of the compilation of the co-located workshops on DSM'11, TMC'11, AGERE!'11, AOOPES'11, NEAT'11, \& VMIL'11, SPLASH '11 Workshops, pages 7-12. ACM, 2011. ISBN 978-1-4503-1183-0. URL http://doi.acm.org/10.1145/2095050.2095053.

[12] A. Ledeczi, M. Maroti, A. Bakay, G. Karsai, J. Garrett, C. Thomason, G. Nordstrom, J. Sprinkle, and P. Volgyesi. The Generic Modeling Environment. In Workshop on Intelligent Signal Processing, 2001. .

[13] D. D. F. Marcos, B. Jean, J. Frédéric, B. Erwan, and G. Guillaume. AMW: A generic model weaver. In Proc. of the lères Journées sur l'Ingénierie Dirigée par les Modèles, volume 200. Citeseer, 2005.

[14] S. Melnik, E. Rahm, and P. A. Bernstein. Rondo: A programming platform for generic model management. In Proceedings of the 2003 ACM SIGMOD international conference on Management of data, page 193-204. ACM, 2003.

[15] R. J. Miller, M. A. Hernández, L. M. Haas, L.-L. Yan, C. H. Ho, R. Fagin, and L. Popa. The clio project: managing heterogeneity. SIgMOD Record, 30:78-83, 2001.

[16] B. Omelayenko. RDFT: A mapping meta-ontology for business integration. In Proc. of the Workshop on Knowledge Transformation for the Semantic Web at the 15th European Conference on Artificial Intelligence (KTSW2002), page 77-84, 2002.

[17] Sirius. URL http://www.eclipse.org/sirius/.

[18] D. Steinberg, F. Budinsky, M. Paternostro, and E. Merks. EMF: Eclipse Modeling Framework. The Eclipse Series. Addison-Wesley, 2nd edition, December 2008. ISBN 01311425420.

[19] The Standard Widget Toolkit (SWT). URL http://www.eclipse.org/swt/.

[20] M. Wimmer. From mining to mapping and roundtrip transformations-a systematic approach to model-based tool integration. $\mathrm{PhD}$ thesis, Vienna University of Technology, 2008.

[21] Xtend. URL http://www.eclipse.org/xtend/. 